TRANSACTIONS OF THE

AMERICAN MATHEMATICAL SOCIETY

Volume 361, Number 3, March 2009, Pages 1351-1369

S 0002-9947(08)04658-8

Article electronically published on October 17, 2008

\title{
RADON TRANSFORM ON SYMMETRIC MATRIX DOMAINS
}

\author{
GENKAI ZHANG
}

\begin{abstract}
Let $\mathbb{K}=\mathbb{R}, \mathbb{C}, \mathbb{H}$ be the field of real, complex or quaternionic numbers and $M_{p, q}(\mathbb{K})$ the vector space of all $p \times q$-matrices. Let $X$ be the matrix unit ball in $M_{n-r, r}(\mathbb{K})$ consisting of contractive matrices. As a symmetric space, $X=G / K=O(n-r, r) / O(n-r) \times O(r), U(n-r, r) / U(n-r) \times U(r)$ and respectively $S p(n-r, r) / S p(n-r) \times S p(r)$. The matrix unit ball $y_{0}$ in $M_{r^{\prime}-r, r}$ with $r^{\prime} \leq n-1$ is a totally geodesic submanifold of $X$ and let $Y$ be the set of all $G$-translations of the submanifold $y_{0}$. The set $Y$ is then a manifold and an affine symmetric space. We consider the Radon transform $\mathcal{R} f(y)$ for functions $f \in C_{0}^{\infty}(X)$ defined by integration of $f$ over the subset $y$, and the dual transform $\mathcal{R}^{t} F(x), x \in X$ for functions $F(y)$ on $Y$. For $2 r<n, 2 r \leq r^{\prime}$ with a certain evenness condition in the case $\mathbb{K}=\mathbb{R}$, we find a $G$-invariant differential operator $\mathcal{M}$ and prove it is the right inverse of $\mathcal{R}^{t} \mathcal{R}, \mathcal{R}^{t} \mathcal{R} \mathcal{M} f=c f$, for $f \in C_{0}^{\infty}(X), c \neq 0$. The operator $f \rightarrow \mathcal{R}^{t} \mathcal{R} f$ is an integration of $f$ against a (singular) function determined by the root systems of $X$ and $y_{0}$. We study the analytic continuation of the powers of the function and we find a BernsteinSato type formula generalizing earlier work of the author in the set up of the Berezin transform. When $X$ is a rank one domain of hyperbolic balls in $\mathbb{K}^{n-1}$ and $y_{0}$ is the hyperbolic ball in $\mathbb{K}^{r^{\prime}-1}, 1<r^{\prime}<n$ we obtain an inversion formula for the Radon transform, namely $\mathcal{M R}^{t} \mathcal{R} f=c f$. This generalizes earlier results of Helgason for non-compact rank one symmetric spaces for the case $r^{\prime}=n-1$.
\end{abstract}

\section{INTRODUCTION}

Let $\mathbb{K}$ be the field of the real, complex and quaternionic numbers. In this paper we will study the Radon transform on non-compact symmetric domains of ( $n-$ $r) \times r$ matrices over $\mathbb{K}$. To motivate and formulate our results recall first the Radon transform on compact Grassmannian manifolds. Consider the Grassmannian manifold $X_{c}=G(n, r)$ of $r$-dimensional subspaces in $\mathbb{K}^{n}$. Let $1 \leq r<r^{\prime}<n$. The Radon transform $\phi(y)=\mathcal{R} f(y), y \in G\left(n, r^{\prime}\right)$, of $f(x)$ on $G(n, r)$ is defined as the integration over the subset of all subspaces $x \in G(n, r)$ which are contained in the fixed subspace $y \in Y_{c}=G\left(n, r^{\prime}\right)$. The dual Radon transform $\left(\mathcal{R}^{t} \phi\right)(x)$ for a function $\phi(y)$ on $G\left(n, r^{\prime}\right)$ is defined as an integration over the subset of all $y \in G\left(n, r^{\prime}\right)$ which

Received by the editors January 17, 2007.

2000 Mathematics Subject Classification. Primary 22E45, 33C67, 43A85, 44A12.

Key words and phrases. Radon transform, inverse Radon transform, symmetric domains, Grassmannian manifolds, Lie groups, fractional integrations, Bernstein-Sato formula, Cherednik operators, invariant differential operators.

This research was supported by the Swedish Science Council (VR). 
contain the given subspace $x \in G(n, r)$. One natural question is to invert the Radon transform. To do that one observes first that the space $X_{c}=G(n, r)$ is a compact symmetric space $X_{c}=G_{c} / K, G_{c}=U(n ; \mathbb{K}), K=U(r ; \mathbb{K}) \times U(n-r ; \mathbb{K})$ and that the operator $\mathcal{R}^{t} \mathcal{R}$ is defined on $X_{c}$ and is $G_{c}$-invariant. The $L^{2}$-space $L^{2}\left(X_{c}\right)$ of functions on $X_{c}$ is decomposed as a direct sum of irreducible subspaces of $G_{c}$ with multiplicity free by the Cartan-Helgason theorem. In his paper 9] Grinberg computes the eigenvalues of $\mathcal{R}^{t} \mathcal{R}$ on the irreducible subspaces. (More precisely the operator $\mathcal{R}$ maps highest weight vectors to highest weight vectors in the respective function spaces on $X_{c}$ and on $Y_{c}$, and Grinberg computes the eigenvalue of $\mathcal{R}$ under some suitable normalization, which then gives the eigenvalues of $\mathcal{R}^{t} \mathcal{R}$.) For the complex, quaternionic and real Grassmannian with $\operatorname{rank}(X) \leq \operatorname{rank}\left(G\left(n, r^{\prime}\right)\right)$ and certain even conditions in the real case it turns out that there exists an invariant differential operator $\mathcal{M}$ which is the left inverse of $\mathcal{R}^{t} \mathcal{R}$, namely $\mathcal{M} \mathcal{R}^{t} \mathcal{R} f=f$ for smooth functions $f$; thus $\mathcal{M R}^{t}$ is the left inverse of $\mathcal{R}$ giving an inversion formula for $\mathcal{R}$. Some different inversion formulas had also been studied earlier; see [6] and the references therein.

Recently some explicit constructions of the invariant differential operator have been studied; see e.g. [17 and 7 . It is a natural and very interesting question to invert the Radon transform on non-compact symmetric spaces, namely replacing the compact spaces $X_{c}$ and $Y_{c}$ by their non-compact duals $X$ and $Y$.

When $r=1$ the space $G(n, 1)$ is the projective space $P^{n-1}(\mathbb{K})$, and its noncompact dual space $X=G / K$ can be realized as the unit ball in $\mathbb{K}^{n-1}$. The unit ball $y_{0}$ in $\mathbb{K}^{r^{\prime}-1}$ can be realized as a totally geodesic submanifold of $X$. We let $Y$ be the set of all $G$-translates of $y_{0} ; Y$ is then an affine symmetric space and is in particular an analytic manifold. In [13, Chapter I] Helgason studied the Radon transform from functions on $X$ to $Y$ for the case when $r^{\prime}=n-1$ and found an inversion formula by a case by case computation; in the special case of real hyperbolic spaces in $\mathbb{R}^{n-1}$ he has also found an inversion formula for any $r^{\prime} \leq n-1$; see [14, Chapter III, Theorem 1.5], and [21.

The Radon transform can also be defined on higher rank non-compact symmetric spaces by integration over totally geodesic submanifolds, but finding its inversion has become rather complicated, for several reasons. Firstly the decomposition of $L^{2}(X)$ under $G$ is a direct integral (rather than the direct sum) and the operator $\mathcal{R}^{t} \mathcal{R}$ is $G$-invariant but not bounded, so that integration against the spherical functions is analytically complicated, compared with the compact case 9. Secondly, in contrast to the rank one case [13, Chapter I] it is not clear if one can find an invariant differential operator as a left inverse of the operator $\mathcal{R}^{t} \mathcal{R}$, because one does not know if the operator $\mathcal{R}^{t} \mathcal{R}$ commutes with invariant differential operators (and we believe it does not); if, on the other hand, we want to find a right inverse of $\mathcal{R}^{t} \mathcal{R}$, then the analytic continuation of the integration defining $\mathcal{R}^{t} \mathcal{R}$ is difficult to handle since generally speaking no explicit construction of invariant differential operators is given.

In the present paper we will study the Radon transform on the non-compact dual $X=G / K$ of the Grassmannian manifold $X_{c}=G_{c} / K$, with $G=U(n-r, r ; \mathbb{K})$. The space $X$ can be realized as the matrix unit ball of $(n-r) \times r$ matrices. There is a natural totally geodesic submanifold $y_{0}$ consisting of $\left(r^{\prime}-r\right) \times r$ matrices in $X$. The set of all $G$-translates of $y_{0}$ is a manifold $Y$, and is an affine symmetric space

$$
Y=G / H, \quad H=U\left(n-r^{\prime} ; \mathbb{K}\right) \times U\left(r^{\prime}-r, r ; \mathbb{K}\right)
$$


of rank $\min \left\{r^{\prime}, n-r^{\prime}\right\}$. We assume

$$
2 r \leq \min \left\{r^{\prime}, 2\left(n-r^{\prime}\right)\right\}
$$

so that the submanifold $y_{0}$ is of the same rank as $X$, which in turn is less than that of $Y$.

The transform $\mathcal{R}^{t} \mathcal{R}$ on the space $C_{0}^{\infty}(X)$ is $G$-invariant. The value of $\mathcal{R}^{t} \mathcal{R} f(x)$ at $x=o$ is an integration of $f$ against a certain power of the hyperbolic sine function $|\mathrm{SH}(t)|^{\delta}$ (see the formula (2.15)) determined by the root systems of $y_{0}$ and $X$. We study thus the meromorphic continuation $|\mathrm{SH}(t)|^{\delta}$ in $\delta \in \mathbb{C}$. For that purpose we find a certain Bernstein-Sato type formula (see Theorem 3.1) for the function $|\mathrm{SH}(t)|^{\delta}$ using the Cherednik operators for general root multiplicities, which generalizes an earlier formula for the function $|\mathrm{CH}(t)|^{\delta}$ in the context of the Berezin transform $([24,25])$ and which might be of independent interest. The meromorphic continuation turns out to be rather complicated, and by using our Bernstein-Sato formula and earlier work of Gindikin on Riesz type distributions we are able to prove that by proper normalization it has analytic continuation and that it gives the Dirac delta distribution at the base point; see Theorem 3.4 and Proposition 4.2. We can then find the right inverse of the transform $\mathcal{R}^{t} \mathcal{R}$, proving that

$$
\mathcal{R}^{t} \mathcal{R} \mathcal{M} f=c f,
$$

where $\mathcal{M}$ is an invariant differential operator on $X$ given in terms of the Cherednik operators (and we can thus find its eigenvalues on the spherical functions, namely its image under the Harish-Chandra homomorphism).

In the case of rank one domains mentioned above with $X$ being the unit ball in $\mathbb{K}^{n-1}$ and $y_{0} \subset X$ the ball in $\mathbb{K}^{r^{\prime}-1}$, we can also include the exceptional rank one domain into our consideration, namely $X$ being the unit ball in $\mathbb{O}^{2}$ and $y_{0}$ the unit ball in $\mathbb{O}=\mathbb{R}^{8}$. In this case we find an inversion formula for the Radon transform due to the commutativity of $\mathcal{M}$ with $\mathcal{R}^{t} \mathcal{R}$. We give a systematic treatment for all cases. So our result generalizes that of Helgason for $r^{\prime}=n-1$. To our knowledge our results are new also in the rank one case.

The author would like to thank the organizers of the workshop "Complex Analysis, Operator Theory and Mathematical Physics" at the Erwin Schrödinger Institute for the invitation and the institute for its support and hospitality. The author also thanks Professors C. Dunkl, H. Schlichtkrull and H. Upmeier for some helpful correspondence and discussions.

\section{SYMMETRIC MATRIX DOMAINS AND RADON TRANSFORM}

In this section we introduce the symmetric domain $X$, the Radon transform $\mathcal{R}$ and the dual Radon transform $\mathcal{R}^{t}$.

2.1. The matrix domain $X$. The matrix domain $X$ can be introduced in the general framework of Jordan triples [18], or symmetric spaces ([13] and [15]). We recall briefly some necessary and elementary results.

Let $\mathbb{K}=\mathbb{R}, \mathbb{C}, \mathbb{H}$ be the field of real, complex or quaternionic numbers with real dimension

$$
a:=\operatorname{dim}_{\mathbb{R}} \mathbb{K}=1,2,4
$$

and let $x \rightarrow \bar{x}$ be the standard conjugation. Let $M_{l, k}:=M_{l, k}(\mathbb{K})$ be the space of $n \times k$ matrices with entries $x$ in $\mathbb{K}$. For brevity we denote (with some abuse of 
notation) by $U(l, k)=U(l, k ; \mathbb{K})$ the group of $\mathbb{K}$-linear transformations $g$ of $\mathbb{K}^{l+k}$ preserving the following quadratic form in $\mathbb{K}^{l+k}$ :

$$
\bar{x}_{1} x_{1}+\cdots+\bar{x}_{l} x_{l}-\bar{x}_{l+1} x_{l+1}-\cdots-\bar{x}_{l+k} x_{l+k} .
$$

Here by a $\mathbb{K}$-linear transformation $g$ we mean $g(v c)=g(v) c, v \in \mathbb{K}^{k}, c \in \mathbb{K}$. Then $U(l, k)$ is the classical group $O(l, k), U(l, k), S p(l, k)$ accordingly.

We consider, for $1 \leq r<n$, the domain

$$
X=\left\{x \in M_{n-r, r} ; I_{r}-x^{*} x>0\right\} .
$$

Here by $I_{r}-x^{*} x>0$ we mean that $I_{r}-x^{*} x$ is a positive $\mathbb{R}$-linear transform of the real Hilbert space $\mathbb{K}^{r}=\mathbb{R}^{d r}$ (with the Euclidean metric $x \rightarrow x^{*} x$ ).

We fix the zero $(n-r) \times r$ matrix as a reference point in $X$ and denote it by $o$. The domain $X$ is a realization of the symmetric space $G / K$,

$$
X=G \cdot o=G / K, \quad G=U(n-r, r), \quad K=U(n-r) \times U(r) .
$$

The group action will be denoted by $g x$ or $g \cdot x$. The domain $X$ can also be characterized using the Jordan triple structure $\{x \bar{y} z\}=x y^{*} z+z y^{*} x$ of $M_{n-r, r}$; see 18 .

Let $\mathfrak{g}$ be the Lie algebra of $U(n-r, r)$ and $\mathfrak{g}=\mathfrak{k}+\mathfrak{p}$ the Cartan decomposition. The space $\mathfrak{p}$ consists of $n \times n=(n-r+r) \times(n-r+r)$ block matrices of the form

$$
\left[\begin{array}{cc}
0_{n-r, n-r} & x \\
x^{*} & 0_{r, r}
\end{array}\right], \quad x \in M_{n-r, r},
$$

and each of them will be identified with the upper left block matrix $x \in M_{n-r, r}$. Here $0_{k, l}$ stands for the zero $k \times l$ matrix.

Assume $r \leq n-r$. The space $X$ is then of rank $r$. Let $\mathfrak{a} \subset \mathfrak{p}=M_{n-r, r}$ be the subspace consisting of matrices $x$ of the form

$$
x=\left[\begin{array}{c}
0_{n-2 r, r} \\
\operatorname{diag}\left\{t_{1}, t_{2}, \cdots, t_{r}\right\}
\end{array}\right]=t_{1} \xi_{1}+\cdots+t_{r} \xi_{r}, \quad t_{j} \in \mathbb{R},
$$

where $\operatorname{diag}\left\{t_{1}, t_{2}, \cdots, t_{r}\right\}$ is the diagonal $r \times r$ matrix with diagonal entries $t_{1}, t_{2}$, $\cdots, t_{r}$ and where the basis vectors $\xi_{1}, \cdots, \xi_{r}$ are self-defined by the formula. We identify $\mathfrak{a}$ with $\mathbb{R}^{r}$ under the basis. Let $\left\{\xi_{j}^{*}\right\}$ be the basis of $\mathfrak{a}^{*}$ dual to $\left\{\xi_{j}\right\}$, $\xi_{j}^{*}\left(\xi_{j}\right)=\delta_{j, k}$. The root system $\Sigma(\mathfrak{g}, \mathfrak{a})$ of $\mathfrak{a}$ in $\mathfrak{g}$ is of type $B$ or $D$ if $\mathbb{K}=\mathbb{R}$ or type $B C$ if $\mathbb{K}=\mathbb{C}, \mathbb{H}$,

$$
\Sigma(\mathfrak{g}, \mathfrak{a})=\left\{ \pm \xi_{j}^{*} \pm \xi_{k}^{*}, j \neq k\right\} \cup\left\{ \pm \xi_{k}^{*}\right\} \cup\left\{ \pm 2 \xi_{k}^{*}\right\}
$$

with multiplicities $a, a(n-2 k)$ and $a-1$ for the respective sets of roots. It is understood that the roots with multiplicity zero will not appear (e.g if $a=1$, the third set is empty).

We denote by $W$ the Weyl group of the root system and fix an ordering so that $\xi_{1}^{*}>\cdots>\xi_{r}^{*}$ for type $B$ or $D$ and $\xi_{1}^{*}>\cdots>\xi_{r}^{*}>0$ for type $B C$.

Let $A=\exp (\mathfrak{a}) \subset G$. Then $A \cdot o$ is a flat submanifold of $X$,

$$
A \cdot o=\left\{\left[\begin{array}{c}
0_{n-2 r, r} \\
\operatorname{diag}\left\{\tanh t_{1}, \cdots, \tanh t_{r}\right\}
\end{array}\right] ; t \in \mathbb{R}^{r}\right\} .
$$

Under the Cartan decomposition $X=K A \cdot o$ the $G$-invariant Riemannian measure on $X$ is (up to constants) given by

$$
\int_{X} f(x) d \mu(x)=\int_{K} \int_{\mathbb{R}^{r}} f\left(k \exp \left(\exp \left(t_{1} \xi_{1}+\cdots+t_{r} \xi_{r}\right) \cdot o\right)\right) d \mu(t) d k,
$$


where (some slight abuse of the notation $d \mu$ )

$$
\begin{aligned}
d \mu(t) & =\prod_{\alpha \in \Sigma_{+}}|2 \operatorname{sh}(\alpha(t))|^{k_{\alpha}} d t \\
& =\prod_{j=1}^{r}\left|2 \operatorname{sh}\left(2 t_{j}\right)\right|^{a-1}\left|2 \operatorname{sh}\left(t_{j}\right)\right|^{a(n-2 r)} \prod_{i<j}\left|2 \operatorname{sh}\left(t_{i}\right) \pm \operatorname{sh}\left(t_{j}\right)\right|^{a} d t_{1} \cdots d t_{r},
\end{aligned}
$$

and $d k$ is the normalized Haar measure on $K$; see [15, Chapter I, Section 5].

2.2. The space $Y$ of totally geodesic submanifolds. We assume throughout the rest of the paper that the rank condition (1.1) holds. We will view the matrix space $M_{r^{\prime}-r, r}$ as a subspace of $M_{n-r, r}$ by identifying each $x \in M_{r^{\prime}-r, r}$ with the matrix $\left[\begin{array}{l}0 \\ x\end{array}\right]$ in $M_{n-r, r}$.

Let $y_{0}$ be the following submanifold of $X$ :

$$
y_{0}=X \cap M_{r^{\prime}-r, r}=\left\{\left[\begin{array}{l}
0 \\
x
\end{array}\right] ; x \in M_{r^{\prime}-r, r}, x^{*} x<I_{r}\right\} .
$$

Then $y_{0}$ is a totally geodesic submanifold of $X$ since $M_{r^{\prime}-r, r}$ is a Jordan subtriple of $M_{n-r, r}$; see [18. It is itself a symmetric domain

$$
y_{0}=G_{0} / K_{0}, \quad G_{0}=U\left(r^{\prime}-r, r\right), \quad K_{0}=U\left(r^{\prime}-r\right) \times U(r)
$$

where $U\left(r^{\prime}-r, r\right)$ is also realized as a subgroup of $G$ by identifying $g \in U\left(r^{\prime}-r, r\right)$ with $\operatorname{diag}\left\{I_{n-r^{\prime}}, g\right\} \in G$. By our assumption (1.1) $y_{0}$ is also of rank $r$. Let $\mathfrak{g}_{0}=$ $\mathfrak{k}_{0}+\mathfrak{p}_{0}$ be the Cartan decomposition of the Lie algebra $\mathfrak{g}_{0}$ of $G_{0}$. The space $\mathfrak{a} \subset \mathfrak{p}$ is also a maximal abelian subspace of $\mathfrak{p}_{0}$ and the root system $\Sigma\left(\mathfrak{g}_{0}, \mathfrak{a}\right)$ is of the same type as that of $\Sigma(\mathfrak{g}, \mathfrak{a})$ in (2.2) as a union of three sets,

$$
\Sigma\left(\mathfrak{g}_{0}, \mathfrak{a}\right)=\left\{ \pm \xi_{j}^{*} \pm \xi_{k}^{*}, j \neq k\right\} \cup\left\{ \pm \xi_{k}^{*}\right\} \cup\left\{ \pm 2 \xi_{k}^{*}\right\}
$$

with respective multiplicities $a, a\left(r^{\prime}-2 r\right), a-1$.

We let

$$
Y=G \cdot y_{0}=\left\{g y_{0} \subset D ; g \in G\right\}
$$

be the set of all $G$-translations of $y_{0}$. It is elementary to check that the stabilizer of $y_{0}$ is

$$
H=U\left(n-r^{\prime}\right) \times G_{0}=U\left(n-r^{\prime}\right) \times U\left(r^{\prime}-r, r\right) .
$$

Thus $Y=G / H$ is an affine symmetric space of rank $\min \left\{r^{\prime}, n-r^{\prime}\right\}$ and is in particular an analytic manifold.

2.3. Radon transform. We first fix the normalization of the $G_{0}$-invariant measure $d \mu_{0}$ on the Riemannian symmetric space $y_{0}$. Under the Cartan decomposition of $y_{0}, y_{0}=K_{0} A \cdot o$, we have

$$
\int_{y_{0}} f(x) d \mu_{0}(x)=\int_{K_{0}} \int_{\mathfrak{a}} f\left(k \exp \left(t_{1} \xi_{1}+\cdots+t_{r} \xi_{r}\right) \cdot o\right) d \mu_{0}(t) d k,
$$

where $d \mu_{0}(t)$ is given as in (2.4) for $d \mu(t)$ with the multiplicity $a(n-2 r)$ replaced by $a\left(r^{\prime}-2 r\right)$, viz.

$$
d \mu_{0}(t)=\prod_{j=1}^{r}\left|2 \operatorname{sh}\left(2 t_{j}\right)\right|^{a-1}\left|2 \operatorname{sh}\left(t_{j}\right)\right|^{a\left(r^{\prime}-2 r\right)} \prod_{i<j}\left|2 \operatorname{sh}\left(t_{i}\right) \pm \operatorname{sh}\left(t_{j}\right)\right|^{a} d t_{1} \cdots d t_{r}
$$


For any $y=g \cdot y_{0} \in Y$ there is a corresponding Riemannian measure $d \mu_{y}$ on $y$ via the isometric mapping $g: y_{0} \rightarrow y$; it is independent of the representatives $g$ by the invariance by $d \mu_{0}$ under the stabilizer $H$ of $y_{0}$.

We define the Radon transform of $\mathcal{R} f$ for a function $f$ on $X$ by

$$
\mathcal{R} f(y)=\int_{y} f(x) d \mu_{y}(x), \quad y \in Y
$$

whenever the integral is absolutely convergent.

To define the dual Radon transform we claim first that

$$
\{y \in Y ; o \in y\}=K \cdot y_{0}:=\left\{k \cdot y_{0} ; k \in K\right\} .
$$

Indeed, it is clear that $o=k \cdot o \in k \cdot y_{0}$ for any $k \in K$ so that $K \cdot y_{0} \subset\{y \in Y ; o \in y\}$. Conversely suppose $o \in y$. Write $y=g \cdot y_{0}$. Thus $o=g z$ for some $z \in y_{0}$ which in turn can be written as $z=g_{z} \cdot o$ for $g_{z} \in G_{0}$. We have then $o=g z=g g_{z} \cdot o$, and that $g g_{z}=k$ for some $k \in K$, namely $g=k g_{z}^{-1}$ and $y=g \cdot y_{0}=k g_{z}^{-1} \cdot y_{0}=k y_{0} \in K \cdot y_{0}$, proving the claim. By the homogeneity of $X=G / K$ we have

Lemma 2.1. For each $x \in X$ we have

$$
Y_{x}:=\{y \in Y ; x \in y\}=g_{x} K \cdot y_{0}:=\left\{g_{x} k \cdot y_{0} ; k \in K\right\},
$$

where $g_{x} \in G$ is such that $x=g_{x} \cdot o$.

The above lemma can also be explained by using the double filtration in [15. In terms of the above lemma we can define the dual Radon transform for a smooth function $\psi$ on $Y$ by

$$
\mathcal{R}^{t} \psi(x)=\int_{K} \psi\left(g_{x} k y_{0}\right) d k, \quad x \in X,
$$

where $x=g_{x} \cdot o$. The function $\mathcal{R}^{t} \psi$ is clearly well defined since it is an integration of a smooth function over compact manifolds, and it can also be written as

$$
\mathcal{R}^{t} \psi(x)=\int_{Y_{x}} \psi(y) d \nu_{x}(y), \quad x \in X,
$$

where $d \nu_{x}(y)$ is some probability measure on $Y_{x}$.

The operator $\mathcal{R}^{t} \mathcal{R}: C_{0}^{\infty}(X) \rightarrow C^{\infty}(X)$ is then

$$
\left(\mathcal{R}^{t} \mathcal{R}\right) f(x)=\int_{K} \int_{y_{0}} f\left(g_{x} k \eta\right) d \mu_{0}(\eta) d k, \quad x=g_{x} \cdot o \in X .
$$

By definition the operators $\mathcal{R}, \mathcal{R}^{t}$ and $\mathcal{R}^{t} \mathcal{R}$ are all $G$-invariant with respect to the corresponding actions of $G$; in particular, we have

$$
\left(\mathcal{R}^{t} \mathcal{R}\right) f(g x)=\left(\mathcal{R}^{t} \mathcal{R}\right)(f \circ g)(x)
$$

for any $g \in G$.

We will write $\mathcal{R}^{t} \mathcal{R}$ as an integration on $X$. For notational convenience we introduce

$$
\mathrm{SH}(t)=\prod_{j=1}^{r} \operatorname{sh} t_{j}, \quad \mathrm{CH}(t)=\prod_{j=1}^{r} \operatorname{ch} t_{j}, \quad t=t_{1} \xi_{1}+\cdots+t_{r} \xi_{r} \in \mathfrak{a}
$$

and with some abuse of notation we denote also by $|\mathrm{SH}(x)|$ and $\mathrm{CH}(x)$ the corresponding $K$-invariant function on $X$. 
Lemma 2.2. The operator $\mathcal{R}^{t} \mathcal{R}$ is given by

$$
\mathcal{R}^{t} \mathcal{R} f(x)=2^{r a\left(r^{\prime}-n\right)} \int_{X}|\mathrm{SH}(\xi)|^{a\left(r^{\prime}-n\right)} f\left(g_{x} \cdot \xi\right) d \mu(\xi), x=g_{x} \cdot o \in X, f \in C_{0}^{\infty}(X) .
$$

Proof. We apply the integral formula (2.9) to (2.14),

$$
\begin{aligned}
\left(\mathcal{R}^{t} \mathcal{R}\right) f(x) & =\int_{K} \int_{K_{0}} \int_{\mathfrak{a}} f\left(g_{x} k k_{0} \exp (t) \cdot o\right) d \mu_{0}(t) d k d k_{0} \\
& =\int_{K} \int_{\mathfrak{a}} f\left(g_{x} k \exp (t) \cdot o\right) d \mu_{0}(t) d k .
\end{aligned}
$$

The measure $d \mu_{0}$ on $\mathfrak{a}$ differs from $d \mu$ by a factor,

$$
d \mu_{0}(t)=2^{r a\left(r^{\prime}-n\right)}|\mathrm{SH}(t)|^{a\left(r^{\prime}-n\right)} d \mu(t)
$$

so that the integration can be written as on $X$ by the Cartan decomposition (2.3) of $X$,

$$
\left(\mathcal{R}^{t} \mathcal{R}\right) f(x)=2^{r a\left(r^{\prime}-n\right)} \int_{X}|\mathrm{SH}(\xi)|^{a\left(r^{\prime}-n\right)} f\left(g_{x} \xi\right) d \mu(\xi)
$$

\section{Bernstein-Sato type formula for the Function $|\mathrm{SH}(t)|^{\delta}$}

3.1. Invariant differential operators on $X$. We recall briefly the relation between invariant differential operators on $X$ and Cherednik operators on $\mathfrak{a}$, the latter being used in the next subsection. The results are well known; see e.g. [10], 19], 12 and 11 .

Let $\mathcal{Q}$ be an invariant differential operator on $X$. If $f$ is a $K$-invariant function on $X$, then so is $\mathcal{Q} f$, and there is a Weyl group $W$-invariant differential operators $\operatorname{rad}(\mathcal{Q})$ on $\mathfrak{a}$ such that $\left.(\mathcal{Q} f)\right|_{\exp \mathfrak{a} \cdot o}=\left.\operatorname{rad}(\mathcal{Q}) f\right|_{\exp \mathfrak{a} \cdot o \text {. }}$ Let $\left\{D_{j}\right\}$ be the Cherednik operator on $\mathfrak{a}$ for the root system $\Sigma(\mathfrak{g}, \mathfrak{a})$; see below. Then there is a Weyl group of $W$-invariant polynomials $p$ so that

$$
\operatorname{rad}(\mathcal{Q})=p\left(D_{1}, \cdots, D_{r}\right)
$$

In this sense the invariant differential operator $\mathcal{Q}$ is uniquely determined by the polynomial $p$ and vice versa. In particular the Harish-Chandra homomorphism of $\mathcal{Q}$ (namely its eigenvalue on spherical functions) is just $p$.

3.2. Bernstein-Sato type formula on a. In this subsection we will prove a certain Bernstein-Sato type formula for the function $\mathrm{SH}(t)$ on $\mathbb{R}^{r}$. We consider a root system $\Sigma$ of type $B, D$ or $B C$ as in Section 2 with general root multiplicity $a$, $2 b$ and $\iota$. The multiplicities of the root system $\Sigma=\Sigma(\mathfrak{g}, \mathfrak{a})$ of $X$ correspond to

$$
(a, 2 b, \iota)= \begin{cases}(1, n-2 k, 0), & \mathbb{K}=\mathbb{R}, \\ (2,2(n-2 k), 1), & \mathbb{K}=\mathbb{C}, \\ (4,4(n-2 k), 3), & \mathbb{K}=\mathbb{H} .\end{cases}
$$

We now assume $a, b, \iota \in \mathbb{C}$ and the results in this subsection hold for this general setting. Let $\rho$ be the half sum of positive roots. Then

$$
\rho=\sum_{j=1}^{r} \rho_{j} \xi_{j}^{*}, \quad \rho_{j}=\iota+b+\frac{a}{2}(r-j) .
$$


Let

$$
\begin{aligned}
& D_{j}=\partial_{j}-a \sum_{i<j} \frac{1}{1-e^{-2\left(t_{i}-t_{j}\right)}}\left(1-s_{i j}\right)+a \sum_{j<k} \frac{1}{1-e^{-2\left(t_{j}-t_{k}\right)}}\left(1-s_{j k}\right) \\
& +a \sum_{k \neq j} \frac{1}{1-e^{-2\left(t_{j}+t_{k}\right)}}\left(1-\sigma_{j k}\right)+2 \iota \frac{1}{1-e^{-4 t_{j}}}\left(1-\sigma_{j}\right)+2 b \frac{1}{1-e^{-2 t_{j}}}\left(1-\sigma_{j}\right)-\rho_{j}
\end{aligned}
$$

be the Cherednik operators acting on functions $f(t)$ on $\mathfrak{a}=\mathbb{R}^{r}$. Here $s_{i j}, \sigma_{i j}, \sigma_{i}$ are the elements in the Weyl group, $s_{i j}=(i j)$ being the permutation of $\xi_{i}$ and $\xi_{j}, \sigma_{i j}$ the signed permutation, $\sigma_{i j}\left(\xi_{i}\right)=-\xi_{j}, \sigma_{i j}\left(\xi_{j}\right)=-\xi_{i}$, and $\sigma_{i}$ the reflection $\sigma_{i}\left(\xi_{i}\right)=-\xi_{i}$, all of these mapping $\xi_{k} \rightarrow \xi_{k}$ for $k \neq i, j$; see [19] in the general case and 24] for root systems of Type $B C$. (Note that we are using a different convention of root system here from that in [19]. The roots here are half of those in [19] whereas the multiplicities are twice those there.)

Theorem 3.1. Let $m_{\delta}$ and $\mathcal{M}_{\delta}$ be the following constant and respectively $W$ invariant polynomials of the Cherednik operators $\left\{D_{j}\right\}$,

$$
m_{\delta}=\prod_{j=1}^{r}(\delta+a(j-1))(\delta-1+\iota+2 b+a(r-j)), \quad \mathcal{M}_{\delta}=\prod_{j=1}^{r}\left(D_{j}^{2}-\left(\delta+\rho_{1}\right)^{2}\right), \quad \delta \in \mathbb{C} .
$$

Then the following Bernstein-Sato type identity holds:

$$
\mathcal{M}_{\delta}|\mathrm{SH}(t)|^{\delta}=m_{\delta}|\mathrm{SH}(t)|^{\delta-2}, \quad t=\left(t_{1}, \cdots, t_{r}\right), t_{j} \neq 0 \quad \forall j .
$$

Proof. Following the proof of a Bernstein-Sato type formula (see (3.4) below) for $\mathrm{CH}^{\delta}$ in 24] we factor the operator $\mathcal{M}_{\delta}$ as

$$
\mathcal{M}_{\delta}=\prod_{j=1}^{r}\left(D_{j}-\left(\delta+\rho_{1}\right)\right) \prod_{j=1}^{r}\left(D_{j}+\delta+\rho_{1}\right),
$$

and consider successively the actions of $\left(D_{j}+\delta+\rho_{1}\right)$ from $j=1$ to $j=r$ and the actions of $\left(D_{j}-\left(\delta+\rho_{1}\right)\right)$ backward from $j=r$ to $j=1$.

Lemma 3.2. For $j=1, \cdots, r$ we have

$$
\begin{aligned}
& \left(\prod_{k=1}^{j}\left(D_{k}+\delta+\rho_{1}\right)\right)|\mathrm{SH}(t)|^{\delta} \\
= & \left(\prod_{k=1}^{j}(\delta+a(k-1))\right)|\mathrm{SH}(t)|^{\delta} \prod_{k=1}^{j}\left(1+\operatorname{coth} t_{k}\right),
\end{aligned}
$$

and

$$
\begin{aligned}
& \left(\prod_{k=j}^{r}\left(D_{k}-\left(\delta+\rho\left(\xi_{1}\right)\right)\right)\right)\left(|\mathrm{SH}(t)|^{\delta} \prod_{i=1}^{r}\left(1+\operatorname{coth} t_{i}\right)\right) \\
= & \left(\prod_{k=j}^{r}(\delta-1+(r-k) a+\iota+2 b)\right)|\operatorname{SH}(t)|^{\delta} \prod_{i=1}^{r}\left(1+\operatorname{coth} t_{i}\right) \prod_{k=j}^{r}\left(\operatorname{coth} t_{k}-1\right),
\end{aligned}
$$

where $t=\left(t_{1}, \cdots, t_{r}\right), t_{k}>0 \forall k$. 
Proof. The first formula can be proved by almost the identical computations as in the proof of Lemmas 2.2 24] changing ch to sh, tanh to coth, producing also the same factor $\prod_{k=1}^{j}(\delta+a(k-1))$. Also the second formula can be proven as in the proof of Lemma 2.3, loc. cit.; however a different factor will appear. We give a sketch of the proof for $j=r$, showing the difference. The rest is done by backward induction and some similar yet long computations. For brevity we denote temporarily $P=\prod_{j=1}^{r}\left(1+\operatorname{coth} t_{j}\right)$ and $\hat{P}_{I}=\prod_{l \notin I}\left(1+\operatorname{coth} t_{l}\right)$ for any index subset $I$. We compute $D_{r}\left(|\mathrm{SH}(t)|^{\delta} P\right)$ and write the result in terms of $|\mathrm{SH}(t)|^{\delta} P$ and $|\mathrm{SH}(t)|^{\delta} P\left(\operatorname{coth} t_{r}-1\right)$. Using

$$
\frac{d}{d s}|\operatorname{sh}(s)|^{\delta}=\delta|\operatorname{sh}(s)|^{\delta}(\operatorname{coth} s-1)+\delta|\operatorname{sh}(s)|^{\delta}, \quad \frac{d}{d s} \operatorname{coth}(s)=-(\operatorname{coth} s+1)(\operatorname{coth} s-1)
$$

and that $P$ is invariant under permutations, we have

$$
\begin{aligned}
& D_{r}\left(|\mathrm{SH}(t)|^{\delta} P\right) \\
& =\delta|\mathrm{SH}(t)|^{\delta} P\left(\operatorname{coth} t_{r}-1\right)+\delta|\mathrm{SH}(t)|^{\delta} P-|\mathrm{SH}(t)|^{\delta} \hat{P}_{r}\left(\operatorname{coth} t_{r}+1\right)\left(\operatorname{coth} t_{r}-1\right) \\
& \quad+2 a|\mathrm{SH}(t)|^{\delta} \sum_{i<r} \hat{P}_{i, r} \frac{e^{t_{i}+t_{r}}}{e^{t_{i}+t_{r}}-e^{-\left(t_{i}+t_{r}\right)}}\left(\operatorname{coth} t_{r}+\operatorname{coth} t_{i}\right) \\
& \quad+2 \iota|\mathrm{SH}(t)|^{\delta} \hat{P}_{r} \frac{e^{2 t_{r}}}{e^{2 t_{r}}-e^{-2 t_{r}}} 2 \operatorname{coth} t_{r}+2 b|\mathrm{SH}(t)|^{\delta} \hat{P}_{r} \frac{e^{t_{r}}}{e^{t_{r}}-e^{-t_{r}}} 2 \operatorname{coth} t_{r} \\
& -\rho_{r}|\mathrm{SH}(t)|^{\delta} P .
\end{aligned}
$$

The second term is

$$
-|\mathrm{SH}(t)|^{\delta} \hat{P}_{r}\left(\operatorname{coth} t_{r}+1\right)\left(\operatorname{coth} t_{r}-1\right)=-|\mathrm{SH}(t)|^{\delta} P\left(\operatorname{coth} t_{r}-1\right) .
$$

Each term in the summation $\sum_{i<r}$ is

$$
\begin{aligned}
& \hat{P}_{i, r} \frac{\left(\operatorname{ch} t_{i}+\operatorname{sh} t_{i}\right)\left(\operatorname{ch} t_{r}+\operatorname{sh} t_{r}\right)}{2 \operatorname{sh}\left(t_{i}+t_{r}\right)}\left(\operatorname{coth} t_{i}+\operatorname{coth} t_{r}\right) \\
= & \frac{1}{2} \hat{P}_{i, r} \frac{\left(\operatorname{ch} t_{i}+\operatorname{sh} t_{i}\right)\left(\operatorname{ch} t_{r}+\operatorname{sh} t_{r}\right)}{\operatorname{sh}\left(t_{i}+t_{r}\right)} \frac{\operatorname{sh}\left(t_{i}+t_{r}\right)}{\operatorname{sh} t_{i} \operatorname{sh} t_{r}} \\
= & \frac{1}{2} \hat{P}_{i, r}\left(\operatorname{coth} t_{r}+1\right)\left(\operatorname{coth} t_{i}+1\right)=\frac{1}{2} P .
\end{aligned}
$$

In the next summand we notice that

$$
\begin{aligned}
& \hat{P}_{r} \frac{e^{2 t_{r}}}{e^{2 t_{r}}-e^{-2 t_{r}}} \operatorname{coth} t_{r}=\hat{P}_{r} \frac{\operatorname{sh}\left(2 t_{r}\right)+\operatorname{ch}\left(2 t_{r}\right)}{2 \operatorname{sh}\left(2 t_{r}\right)} \operatorname{coth} t_{r} \\
& \quad=\hat{P}_{r} \frac{\operatorname{sh}\left(2 t_{r}\right)+\operatorname{ch}\left(2 t_{r}\right)}{2 \operatorname{sh}\left(2 t_{r}\right)} \operatorname{coth} t_{r}=\frac{1}{4} \hat{P}_{r}\left(\operatorname{coth}^{2} t_{r}-1+2\left(\operatorname{coth} t_{r}+1\right)\right) \\
& \quad=\frac{1}{4}\left(P\left(\operatorname{coth} t_{r}-1\right)+2 P\right)
\end{aligned}
$$

Similarly, in the next term we have

$$
P_{r} \frac{e^{t_{r}}}{e^{t_{r}}-e^{-t_{r}}} \operatorname{coth} t_{r}=\frac{1}{2}\left(P\left(\operatorname{coth} t_{r}-1\right)+P\right)
$$


and then

$$
\begin{aligned}
& \left(D_{r}-\left(\delta+\rho_{1}\right)\right)\left(|\mathrm{SH}(t)|^{\delta} P\right) \\
= & \left(\delta+2 \iota+2 b+(r-1) a-\rho_{r}-\left(\delta+\rho_{1}\right)\right)|\mathrm{SH}(t)|^{\delta} P \\
& +(\delta-1+\iota+2 b)|\mathrm{SH}(t)|^{\delta} P\left(\operatorname{coth} t_{r}-1\right),
\end{aligned}
$$

but the first term is vanishing and this proves the identity for $j=r$.

Our result follows then from the first formula for $j=r$ and the second for $j=1$,

$$
\begin{aligned}
\mathcal{M}_{\delta}|\mathrm{SH}(t)|^{\delta}= & \prod_{k=1}^{r}\left(D_{k}-\left(\delta+\rho_{1}\right)\right) \prod_{k=1}^{r}\left(D_{k}+\delta+\rho_{1}\right)|\mathrm{SH}(t)|^{\delta} \\
= & \left(\prod_{k=1}^{r}(\delta+a(k-1))\right) \prod_{k=1}^{r}\left(D_{k}-\left(\delta+\rho_{1}\right)\right)\left(|\mathrm{SH}(t)|^{\delta} \prod_{i=1}^{r}\left(\operatorname{coth} t_{i}+1\right)\right) \\
= & \left(\prod_{k=1}^{r}(\delta+a(k-1))\right)\left(\prod_{k=1}^{r}(\delta-1+(r-k) a+\iota+2 b)\right) \\
& \times|\operatorname{SH}(t)|^{\delta} \prod_{i=1}^{r}\left(\operatorname{coth} t_{i}+1\right) \prod_{k=1}^{r}\left(\operatorname{coth} t_{k}-1\right) \\
= & m_{\delta}|\mathrm{SH}(t)|^{\delta-2} .
\end{aligned}
$$

It is worthwhile to make some remarks on the theorem.

Remark 3.3. In 24] the following identity is proved:

$$
\mathcal{M}_{\delta} \mathrm{CH}(t)^{\delta}=(-1)^{r} \prod_{j=1}^{r}(\delta+a(j-1))(\delta-1+\iota+a(r-j)) \mathrm{CH}(t)^{\delta-2},
$$

which is of fundamental importance in the Berezin transform [25] and in spherical transforms of Jacobi type functions 24. The pair of equalities (3.3) and (3.4) is to be compared with the following pair of simple identities (for the flat rank one case with all root multiplicities being zero)

$$
\left(\frac{d^{2}}{d t^{2}}-\delta^{2}\right)|\operatorname{sh}(t)|^{\delta}=\delta(\delta-1)|\operatorname{sh}(t)|^{\delta-2}, \quad\left(\frac{d^{2}}{d t^{2}}-\delta^{2}\right) \operatorname{ch}(t)^{\delta}=-\delta(\delta-1) \operatorname{ch}(t)^{\delta-2} .
$$

Note that in the flat case for $\delta$ being an even integer it is possible to derive one equality in the pair from the another by using analytic continuation in $t$, the fact that $\operatorname{sh}\left(t+\frac{\pi}{2} i\right)=i \operatorname{ch} t$ and that the operator $\frac{d^{2}}{d t^{2}}$ is translation invariant. However in the non-flat case the two equalities (3.4) and (3.3) are considerably different, even though the methods of the proofs are similar. Firstly the Cherednik operators are not translation invariant, and it seems to the author that the intertwining relations between the Cherednik operators and translations are not fully understood (see [3] for some related questions); secondly the constants in the right hand of (3.5) differ just by a sign change and this is not the case for (3.4) and (3.3) when $b \neq 0$. Also, the equality (3.3) holds true for $t$ with $t_{j} \neq 0$ as the function $|\mathrm{SH}(t)|^{\delta}$ has singularity on the hypersurfaces $t_{j}=0, j=1, \cdots, r$ for general $\delta \in \mathbb{C}$ and is not differentiable, whereas $\mathrm{CH}(t)^{\delta}$ is a real analytic function. 
When the root multiplicities correspond to the Grassmannian manifold in $\mathbb{K}^{n}$ and for certain specific integers $\delta$ (depending on the domain) it should be possible to derive some Bernstein-Sato type formula for the function $\left|\left(\prod \sin t_{j}\right)\right|^{\delta}$ on the compact torus $i \mathbb{R}^{r} / i \pi \mathbb{Z}^{r}$ by combining the result of Grinberg [9] on the Radon transform on $X^{c}$ and the result of Opdam [19] on eigenvalues of the Cherednik operators, and it is interesting to carry out the details; see also [17] and [7].

3.3. The meromorphic continuation of the distribution $|\mathrm{SH}(t)|^{\delta}$ on root systems with positive multiplicities. We assume $\Sigma$ is, as in the previous subsection, a root system with general non-negative multiplicities $a \geq 0, b \geq 0$ and $\iota \geq 0$.

We notice first that, if $f \in C_{0}^{\infty}\left(\mathbb{R}^{r}\right)^{W}$, namely the Weyl group invariant, the integral

$$
\int_{\mathfrak{a}}|\mathrm{SH}(t)|^{\delta} f(t) d \mu(t)
$$

is absolutely convergent if $\Re(\delta)>-1-l-2 b$, and it defines a distribution analytic in $\delta$. We consider now the integral

$$
\zeta_{\delta}(f)=\frac{1}{z_{\delta}} \int_{\mathfrak{a}}|\mathrm{SH}(t)|^{\delta} f(t) d \mu(t)
$$

where

$$
z_{\delta}=\Gamma_{a}\left(\frac{\delta}{2}+\frac{a}{2}(r-1)+1\right) \Gamma_{a}\left(\frac{\delta+\iota+2 b}{2}+\frac{a}{2}(r-1)+1\right)
$$

and

$$
\Gamma_{a}(\lambda)=\prod_{j=1}^{r} \Gamma\left(\lambda-\frac{a}{2}(j-1)\right)
$$

is the Gindikin Gamma function.

Theorem 3.4. The distribution $\zeta_{\delta}$ on $C_{0}^{\infty}\left(\mathbb{R}^{r}\right)^{W}$ for $\Re \delta>-1-\iota-2 b$ has analytic continuation to $\delta \in \mathbb{C}$ and

$$
\mathcal{M}_{\delta} \zeta_{\delta}=2^{2 r} \zeta_{\delta-2}
$$

where $\mathcal{M}_{\delta}$ is the differential operator in Theorem 3.1.

Proof. We rewrite the factor $m_{\delta}$ in Theorem 3.1 using $z_{\delta}$. Changing $j-1$ to $r-j$ we see that

$m_{\delta}=2^{2 r} \prod_{j=1}^{r}\left(\frac{\delta}{2}+\frac{a}{2}(r-1)-\frac{a}{2}(j-1)\right) \prod_{j=1}^{r}\left(\frac{\delta-1+\iota+2 b}{2}+\frac{a}{2}(r-1)-\frac{a}{2}(j-1)\right)$,

and each product is a quotient of the Gindikin Gamma function

$$
\prod_{j=1}^{r}\left(\frac{\delta-1+\iota+2 b}{2}+\frac{a}{2}(r-1)-\frac{a}{2}(j-1)\right)=\frac{\Gamma_{a}\left(\frac{\delta-1+\iota+2 b}{2}+\frac{a}{2}(r-1)+1\right)}{\Gamma_{a}\left(\frac{\delta-1+\iota+2 b}{2}+\frac{a}{2}(r-1)\right)} .
$$

Namely $m_{\delta}=2^{2 r}=2^{2 r} \frac{z_{\delta}}{z_{\delta-2}}$ and the Bernstein-Sato formula (3.3) is then

$$
\frac{1}{z_{\delta}} \mathcal{M}_{\delta}|\mathrm{SH}|^{\delta}=2^{2 r} \frac{1}{z_{\delta-2}}|\mathrm{SH}|^{\delta-2} \text {. }
$$


The functions $|\mathrm{SH}|^{\delta}$ and $\mathcal{M}_{\delta}|\mathrm{SH}|^{\delta}$ for sufficient large $\delta$ are well-defined distributions and are analytic in $\delta$, and so is the constant $\frac{1}{z_{\delta}}$. By partial integration we have

$$
\begin{aligned}
\zeta_{\delta}(f) & =\frac{1}{z_{\delta}} \int_{\mathbb{R}^{r}}|\mathrm{SH}(t)|^{\delta} \mathcal{M}_{\delta} f(t) d \mu(t) \\
& =\frac{1}{z_{\delta}} \int_{\mathbb{R}^{r}} \mathcal{M}_{\delta}|\mathrm{SH}(t)|^{\delta} \mathcal{M} f(t) d \mu(t) \\
& =2^{2 r} \zeta_{\delta-2}(f) ;
\end{aligned}
$$

see the proof of [19, Lemma 7.8]. This proves the analytic continuation.

See also Remark 5.5 for a possible refinement of the above theorem.

\section{The Dirac Distribution as a meromorphic Continuation OF THE DisTRIBUTION $|\mathrm{SH}(t)|^{\delta}$ ON SYMMETRIC DOMAINS}

4.1. Gårding-Gindikin distribution $\left(x_{1} \cdots x_{r}\right)_{+}^{\lambda-\frac{a}{2}(r-1)-1}$ on $\mathbb{R}^{r}$. In this subsection we reformulate the result of Gindikin 44 on Riesz type integrals (also called Gårding-Gindikin distributions) on the Jordan algebra of real symmetric, complex Hermitian and Quaternionic Hermitian $r \times r$ matrices) in polar coordinates with the test function being $U(r, \mathbb{K})$-invariant smooth functions $f$ of compact support. (In [4] the more general case of Schwartz functions is considered.) We will identify such functions $f$ as functions on $\mathbb{R}^{r}$. We refer to [4] for an account of symmetric cones; see also [20]. Let

$$
\left(x_{1} \cdots x_{r}\right)_{+}=\left(x_{1}\right)_{+} \cdots\left(x_{r}\right)_{+}
$$

where $x_{+}$on $\mathbb{R}$ is the function $x_{+}=x$ if $x>0$ and $x_{+}=0$ if $x \leq 0$. Consider the Gårding-Gindikin integral

$$
\begin{aligned}
\mathcal{G}_{\lambda}(f) & =\frac{1}{r !} \frac{1}{\Gamma_{a}(\lambda)} \int_{\mathbb{R}^{r}}\left(x_{1} \cdots x_{r}\right)_{+}^{\lambda-\frac{a}{2}(r-1)-1} f\left(x_{1}, \cdots, x_{r}\right) \prod_{i<j}\left|x_{i}-x_{j}\right|^{a} d x \\
& =\frac{1}{r !} \frac{1}{\Gamma_{a}(\lambda)} \int_{\mathbb{R}_{+}^{r}}\left(x_{1} \cdots x_{r}\right)^{\lambda-\frac{a}{2}(r-1)-1} f\left(x_{1}, \cdots, x_{r}\right) \prod_{i<j}\left|x_{i}-x_{j}\right|^{a} d x,
\end{aligned}
$$

where $f \in C_{0}\left(\mathbb{R}^{r}\right)$, is symmetric in $x_{1}, \cdots, x_{r}$, and $\Gamma_{a}(\lambda)$ is the Gindikin Gamma function (3.8), and $a=1,2,4$.

The following lemma follows immediately from [4, Theorem VII.2.2] and from (a special case of) Chevalley's theorem that the restriction to the diagonal of $U(r, \mathbb{K})$ invariant smooth functions of compact support on the space of all symmetric matrices to the diagonal matrices is an isomorphism (see [15, Chapter II, Section 5] for some general statements).

Lemma 4.1. Suppose $f \in C_{0}^{\infty}\left(\mathbb{R}^{r}\right)$ is symmetric. The distribution $\mathcal{G}_{\lambda}$ has analytic continuation to $\lambda \in \mathbb{C}$ and

$$
\mathcal{G}_{0}=c_{0} \mathcal{I}_{0}
$$

where

$$
c_{0}=\prod_{1 \leq i<i \leq r} \frac{\Gamma\left(\frac{a}{2}(j-i+1)\right)}{\Gamma\left(\frac{a}{2}(j-i)\right)}
$$

and $\mathcal{I}_{0}$ is the Dirac distribution at $x=0$. 
4.2. Analytic continuation of $|\mathrm{SH}(t)|^{\delta}$. The case of symmetric domains. We let $\Sigma$ be as in Section 2 the root system of the symmetric domain $X$ with the multiplicity $(a, 2 b, \iota)$ given as in (3.1).

Proposition 4.2. Suppose that the root multiplicities satisfy $1+\iota+2 b>r(a-1)$. Let $\delta_{0} \in \mathbb{R}$ be such that

$$
-1-\iota-2 b<\delta_{0}<-r(a-1)
$$

and that

$$
l=\frac{\delta_{0}+\iota+2 b-1+a(r-1)}{2}+1
$$

is a positive integer. Then for any $f \in C_{0}^{\infty}(\mathfrak{a})^{W}$, the following formula holds:

$$
\left(\prod_{k=0}^{l-1} \mathcal{M}_{\delta_{0}-2 k}\right)|\mathrm{SH}(t)|^{\delta_{0}}=c_{1} \mathcal{I}_{0}
$$

in the sense of distribution on $C_{0}^{\infty}(\mathfrak{a})^{W}$ (defined by the integration against $d \mu(t)$ ), where $\mathcal{I}_{0}$ is the Dirac distribution at $t=0$,

$c_{1}=2^{r(2 b+2 \iota)+l r} r ! \Gamma\left(\frac{1}{2}\left(\delta_{0}+\iota+2 b-1\right)+\frac{a}{2}(r-1)+1\right)\left(\prod_{k=0}^{l-1} \prod_{j=1}^{r}\left(\delta_{0}-2 k+a(j-1)\right) c_{0}\right.$ and $c_{1} \neq 0$.

Proof. Let $\Re \beta>2 l-\iota-2 b+1$ be sufficiently large. We compute

$$
\begin{aligned}
S_{\beta}(f): & =\int_{\mathbb{R}^{r}}|\mathrm{SH}(t)|^{\beta}\left(\prod_{k=0}^{l-1} \mathcal{M}_{\beta-2 k}\right) f(t) d \mu(t) \\
& =\int_{\mathbb{R}^{r}}\left(\left(\prod_{k=0}^{l-1} \mathcal{M}_{\beta-2 k}\right)|\mathrm{SH}(t)|^{\beta}\right) f(t) d \mu(t) \\
& =\left(\prod_{k=0}^{l-1} m_{\beta-2 k}\right) \int_{\mathbb{R}^{r}}|\mathrm{SH}(t)|^{\beta-2 l} f(t) d \mu(t) \\
& =\left(\prod_{k=0}^{l-1} m_{\beta-2 k}\right) 2^{r} \int_{\mathbb{R}_{+}^{r}}|\mathrm{SH}(t)|^{\beta-2 l} f(t) d \mu(t) .
\end{aligned}
$$

Change variables $x_{j}=\operatorname{sh} t_{j}^{2}$. Then

$$
\begin{aligned}
S_{\beta}(f) & =2^{r(2 b+2 \iota)}\left(\prod_{k=0}^{l-1} m_{\beta-2 k}\right) \int_{\mathbb{R}_{+}^{r}}\left(x_{1} \cdots x_{r}\right)^{\frac{1}{2}(\beta-2 l+\iota+2 b-1)} \\
& \times \prod_{j=1}^{r}\left(1+x_{j}\right)^{\frac{1}{2}(\iota-1)} f\left(x_{1}, \cdots, x_{r}\right) \prod_{i<j}\left(x_{i}-x_{j}\right)^{a} d x_{1} \cdots d x_{r} \\
& =2^{r(2 b+2 \iota)} r !\left(\prod_{k=0}^{l-1} m_{\delta-2 k}\right) \Gamma_{a}\left(\frac{1}{2}(\beta-2 l+\iota+2 b-1)+\frac{a}{2}(r-1)+1\right) \\
& \times \mathcal{G}_{\frac{1}{2}(\beta-2 l+\iota+2 b-1)+\frac{a}{2}(r-1)+1}(F)
\end{aligned}
$$


in terms of the Gårding-Gindikin distribution, where

$$
F\left(x_{1}, \cdots, x_{r}\right)=\prod_{j=1}^{r}\left(1+x_{j}\right)^{\frac{1}{2}(\iota-1)} f\left(x_{1}, \cdots, x_{r}\right)
$$

for $x \in \mathbb{R}_{+}^{r}$ and is extended to any symmetric function in $C_{0}^{\infty}\left(\mathbb{R}^{r}\right)$. Using (3.10) we see that, in the previous formula, the constant in front of the Gårding-Gindikin distribution is (disregarding $2^{r(2 b+2 \iota)} r$ !)

$$
\begin{aligned}
& \left(\prod_{k=0}^{l-1} m_{\delta-2 k}\right) \Gamma_{a}\left(\frac{1}{2}(\beta-2 l+\iota+2 b-1)+\frac{a}{2}(r-1)+1\right) \\
= & 2^{l r} \Gamma_{a}\left(\frac{1}{2}(\beta+\iota+2 b-1)+\frac{a}{2}(r-1)+1\right)\left(\prod_{k=0}^{l-1} \prod_{j=1}^{r}(\delta-2 k+a(j-1))\right)
\end{aligned}
$$

and is analytic for all $\beta \in \mathbb{C}$ such that

$$
\frac{1}{2}(\Re \beta+\iota+2 b-1)+1>0 .
$$

Thus the above formula (4.3) holds for all such $\beta$. Our results follow by taking $\beta=\delta_{0}$ and applying Lemma 4.1. The assumption on the root multiplicities, on $\delta_{0}$ and $l$ guarantees that the constant $c_{1} \neq 0$.

\section{Right inverse of $\mathcal{R}^{t} \mathcal{R}$. Left inverse of $\mathcal{R}$ in the CASE of RANK ONe}

\subsection{Right inverse of the transform $\mathcal{R}^{t} R$.}

Theorem 5.1. Let $X$ be the symmetric domain $X=U(n-r, r ; \mathbb{K}) / U(n-r ; \mathbb{K}) \times$ $U(r, \mathbb{K})$, and $Y=U(n-r, r ; \mathbb{K}) / U\left(n-r^{\prime} ; \mathbb{K}\right) \times U\left(r^{\prime} ; \mathbb{K}\right)$ be the set of $G$-translates of the totally geodesic submanifold $y_{0}$. Suppose that the rank condition (1.1) is satisfied, and that $r^{\prime}-r$ is even when $\mathbb{K}=\mathbb{R}$. Let $\mathcal{M}$ be the invariant differential operator on $X$ so that

$$
\operatorname{rad} \mathcal{M}=\prod_{k=0}^{l-1} \mathcal{M}_{a(r \prime-n)-2 k}, \quad l=\frac{a}{2}\left(r^{\prime}-r\right) .
$$

Then

$$
\mathcal{R}^{t} \mathcal{R} \mathcal{M} f(x)=c f(x), \quad f \in C_{o}^{\infty}(X), \quad x \in X,
$$

where $c=c_{1}$ as given in Proposition 4.2 with $\iota=a-1,2 b=a(n-2 r)$ and $\delta_{0}=a\left(r^{\prime}-n\right)$.

Proof. Suppose first that $f \in C_{0}^{\infty}(X)$ is $K$-invariant. Then by Lemma 2.2,

$$
\left(\mathcal{R}^{t} \mathcal{R} \mathcal{M} f\right)(o)=2^{r a\left(r^{\prime}-n\right)} \int_{\mathfrak{a}}|\mathrm{SH}(t)|^{\delta_{0}} \operatorname{rad}(\mathcal{M}) f(\exp (t) \cdot o) d \mu(t)
$$

with $\delta_{0}=a\left(r^{\prime}-n\right)$. Applying Proposition 4.2 we find

$$
\left(\mathcal{R}^{t} \mathcal{R} \mathcal{M} f\right)(o)=c f(o) .
$$

Now for any $f \in C_{0}^{\infty}(X)$ and for a fixed $x=g_{x} \cdot o$ we let

$$
F(\xi)=\int_{K} f\left(g_{x} k \xi\right) d k
$$


which is a $K$-invariant function in $C_{0}^{\infty}(X)$. The inversion formula for $F$ at $\xi=o$ reads as follows:

$$
\left(R^{t} R \mathcal{M} F\right)(o)=c F(o)=c f(x) .
$$

However by invariance of $R^{t} R$ and $\mathcal{M}$ under $G$ we have

$$
\begin{aligned}
\left(R^{t} R \mathcal{M} F\right)(o) & =\left.\int_{K}\left(\left(R^{t} R \mathcal{M}\right) f\left(g_{x} k \xi\right)\right)\right|_{\xi=o} d k=\left.\int_{K}\left(R^{t} R \mathcal{M} f\right)\left(g_{x} k \xi\right)\right|_{\xi=o} d k \\
& =\int_{K}\left(R^{t} R \mathcal{M} f\right)\left(g_{x} k o\right) d k=\left(R^{t} R \mathcal{M} f\right)(x),
\end{aligned}
$$

completing the proof.

We note that the above theorem is closely related to the surjectivity of the invariant differential operator $\mathcal{M}$. We let $\mathcal{M}$ act on the formula (5.1),

$$
\mathcal{M R}^{t} \mathcal{R} u(x)=c u(x),
$$

where $u=\mathcal{M} f$. Thus we have an inversion formula for functions $u \in C_{0}^{\infty}(X)$ which are in the image of $\mathcal{M}$ on $C_{0}^{\infty}(X)$. Generally speaking, however, the image of an invariant differential operator on $C_{0}^{\infty}(X)$ is not onto. Recall, by a theorem of Helgason [16], that for any $u \in C_{0}^{\infty}(X)$ (and for any invariant differential operator, in particular for $\mathcal{M})$ there is an $f \in C^{\infty}(X)$ such that $\mathcal{M} f=u$; however the function $f$ is not necessarily in $C_{0}^{\infty}(X)$.

5.2. The case of rank one. When $r=1$ the matrix space $M_{1, n-1}(\mathbb{K})=\mathbb{K}^{n-1}$, and the domain $X$ is the hyperbolic ball in $\mathbb{K}^{n-1}$. The subdomain $y_{0}$ in question is the unit ball in $0 \oplus \mathbb{K}^{r^{\prime}-1}$. We consider the Radon transform over the set $Y=G \cdot y_{0}$ of $\left(r^{\prime}-1\right)$-dimensional totally geodesic submanifolds, for any $1<r^{\prime} \leq n-1$. We can also include the exceptional domain into our consideration, with $X$ being the unit ball in $\mathbb{K}^{2}$ where $\mathbb{K}=\mathbb{O}$, the real Cayley numbers, and $y_{0}$ is the unit ball in $0 \oplus \mathbb{O}=\mathbb{R}^{8}, y_{0}=S O_{0}(8,1) / S O(8)$. As symmetric spaces $X=G / K, y_{0}=G_{0} / K_{0}$ with

$$
(\mathfrak{g}, \mathfrak{k})=\left(\mathfrak{f}_{4(-20)}, \mathfrak{s o}(9)\right), \quad\left(\mathfrak{g}_{0}, \mathfrak{k}_{0}\right)=(\mathfrak{s o}(8,1), \mathfrak{s o}(8)) .
$$

The parameters $a, r, r^{\prime}$ are given by

$$
\left(a, r, r^{\prime}\right)=(8,1,2) .
$$

We specialize now the Bernstein-Sato type formula in the rank one cases, with the root multiplicities there given by

$$
(2 b, \iota)=(a(n-2), a-1)
$$

with the convention that $\mathbb{K}^{n-1}=\mathbb{O}^{2}$ and $a(n-2)=8$ when $a=8$ i.e. $\mathbb{K}=\mathbb{O}$. The half sum of the positive roots is $\rho=b+\iota=\frac{a}{2} n-1$.

We normalize the Riemannian metric on $X=G / K$ so that the vector $\xi=\xi_{1} \in \mathfrak{p}$, viewed as a tangent vector of $X$ at $o$, has norm 1 ; we use the same Riemannian measure $d \mu(x)$ as above. The radial part $\operatorname{rad}(\mathcal{L})$ of the Laplace-Beltrami operator $\mathcal{L}$ is then

$$
\operatorname{rad}(\mathcal{L})=D^{2}-\rho^{2}
$$


where $D$ is the Cherednik operator; this follows directly by computing $D^{2}$ and by using the formula in [15, Chapter II] for the radial part of the Laplace-Beltrami operator 1 on the symmetric space $X$.

We let

$$
\lambda_{j}=\left(a(n-1)-a\left(r^{\prime}-1\right)+2(j-1)\right)\left(a\left(r^{\prime}-1\right)+a-2 j\right), \quad j=1,2, \cdots .
$$

Theorem 5.2. Let $\mathbb{K}=\mathbb{R}, \mathbb{C}, \mathbb{H}, \mathbb{O}$ be the ring of real, complex, quaternionic and Cayley numbers. Let $X=G / K$ be the unit ball in $\mathbb{K}^{n-1}$ and $Y=G \cdot y_{0}$ the space of $\left(r^{\prime}-1\right)$-dimensional geodesic submanifolds of $X$. Suppose $r^{\prime}-1$ is even in the real case $\mathbb{K}=\mathbb{R}$ (namely $y_{0}$ is an even $\left(r^{\prime}-1\right)$-dimensional submanifold). Let

$$
l=\frac{a}{2}\left(r^{\prime}-1\right) .
$$

Then the Radon transform $\phi=\mathcal{R} f$, for $f \in C_{0}^{\infty}(X)$, is inverted by

$$
\mathcal{M}\left(\mathcal{R}^{t} \phi\right)=c f,
$$

where

$$
\mathcal{M}=\prod_{j=1}^{l}\left(\mathcal{L}+\lambda_{j}\right)
$$

and $\mathcal{L}$ is the Laplace-Beltrami operator (normalized as above).

Proof. We use Theorem 3.1. The operator $\mathcal{M}_{\delta}$ there is, by (5.2),

$$
\mathcal{M}_{\delta}=D^{2}-(\delta+\rho)^{2}=D^{2}-\rho^{2}+\rho^{2}-(\delta+\rho)^{2}=\operatorname{rad}(\mathcal{L})+(-\delta)(2 \rho+\delta) .
$$

We have

$$
(\mathcal{L}+(-\delta)(2 \rho+\delta))|\mathrm{SH}(x)|^{\delta}=\delta(\delta-1+\iota+2 b)|\mathrm{SH}(x)|^{\delta-2}, \quad x \neq o,
$$

which can also be proved independently by straightforward computations. We recall that $|\mathrm{SH}(x)|$ is extended to a $K$-invariant function on $X$. With this formula it is now easy to study the analytic continuation of the distributions by elementary computations independent of the previous sections. In particular, by Proposition 4.2 we have

$$
\mathcal{M}|\mathrm{SH}(x)|^{a\left(r^{\prime}-n\right)}=c_{3} \mathcal{I}_{o},
$$

where $\mathcal{I}_{o}$ is the Dirac distribution at the origin $o$.

The rest is essentially the same as [15, Chapter I, Section 4] (with some different formulation). Let the operator $\mathcal{M}$ act on the formula $\mathcal{R}^{t} \mathcal{R} f(x)$ in Lemma 2.2 identified as a function of $g \in G$ :

$$
\begin{aligned}
\mathcal{M}_{x} \mathcal{R}^{t} \mathcal{R} f(x) & =\mathcal{M}_{g} \mathcal{R}^{t} \mathcal{R} f(g) \\
& =2^{\left(2 b^{\prime}-2 b\right)} \int_{X}|\mathrm{SH}(\xi)|^{a\left(r^{\prime}-n\right)} \mathcal{M}_{g} f(g \xi) d \mu(\xi) \\
& =2^{\left(2 b^{\prime}-2 b\right)} \int_{X}|\operatorname{SH}(\xi)|^{a\left(r^{\prime}-n\right)}\left(\mathcal{M}_{\xi} f\right)(g \xi) d \mu(\xi) \\
& =2^{\left(2 b^{\prime}-2 b\right)} \int_{X}|\operatorname{SH}(\xi)|^{a\left(r^{\prime}-n\right)}\left(\mathcal{M}_{\xi} f\right)\left(g_{x} \xi\right) d \mu(\xi)
\end{aligned}
$$

\footnotetext{
${ }^{1}$ The Riemann metric used [15] is defined by the Killing form, so that the tangent vector $\xi$ has squared norm $\operatorname{tr}_{\mathfrak{g}}(\operatorname{ad} \xi \operatorname{ad} \xi)=4($ an -2$)$, so that our Laplace-Beltrami operator here is that in [15] multiplied by $4(a n-2)$.
} 
by the bi-invariance of the Laplace-Beltrami operator. The rest follows from (5.4), and the constant $c$ is the same as in Theorem 5.1.

It might be illuminating to write the operator $\mathcal{M}$ as

$$
\mathcal{M}=\prod_{j=1}^{\frac{1}{2} d_{0}}\left(\mathcal{L}+\left(d-d_{0}+2(j-1)\right)\left(d_{0}+a-2 j\right)\right)
$$

where $d=\operatorname{dim}(X)$ and $d_{0}=\operatorname{dim}\left(y_{0}\right)$. The case when $r^{\prime}=n-1$ was proved earlier by Helgason [15] by case by case computations (our $d=\operatorname{dim}(X)$ and $r^{\prime}-1$ corresponds to $n$ and respectively $n-1$ there). The roots of the operator $\mathcal{M}$ as a polynomial of the Laplace-Beltrami operator in the complex case appear also in the context of Helmholtz operators [22]; in particular using our Theorem 3.1 we can get a more precise form of the formula (29) there, but we will go into the details.

Finally we make some remarks on certain interesting open questions.

Remark 5.3. Let $\Sigma$ be a root system with non-negative multiplicities. We introduce the Schwartz space $\mathcal{S}_{\Sigma}^{0}(\mathfrak{a})$ on the root system $\Sigma$ on $\mathfrak{a}$ : A Weyl-group invariant smooth function $f$ on $\mathfrak{a}$ is in $\mathcal{S}_{\Sigma}^{0}(\mathfrak{a})$ if for any $W$-group invariant polynomial $p$,

$$
\sup _{t \in \mathfrak{a}} e^{-N \rho(t)}\left|p\left(D_{1}, \cdots, D_{r}\right) f(t)\right|<\infty
$$

for all $N=0,1, \cdots$. It is easy to see that the function $|\mathrm{SH}(t)|^{\delta}$ can be defined as a distribution on $\mathcal{S}_{\Sigma}^{0}(\mathfrak{a})$ and Theorem 3.4 is still true in this sense.

When the root system corresponds to a Riemannian symmetric space $X=G / K$, this is then the Schwartz $L^{0}$-space $\mathcal{S}^{0}(G)$ of $K$-bi-invariant functions on $G$ [ 5 . Recall [5] that the Schwartz $L^{p}$-space on $X=G / K$ is the space of $K$-invariant smooth functions on $X$ such that for any invariant differential operator $\mathcal{L}$, and any $M>0$,

$$
\sup _{t \in \mathfrak{a}} e^{-(2 / p-2) \rho(t)}\left(1+|t|^{2}\right)^{-M} \phi_{0}(\exp (t) \cdot o)|\mathcal{L} f(\exp (t) \cdot o)|<\infty .
$$

When $p=0$ that condition can be simplified as

$$
\sup _{t \in \mathfrak{a}} e^{-N \rho(t)}|\mathcal{L} f(\exp (t) \cdot o)|<\infty .
$$

To see this, one has to use [5, Theorem 4.6.6] there; see also [1, Proposition 2.2.12]. The last condition is clearly equivalent to (5.5).

Remark 5.4. Note that the question of finding an inversion formula for the Radon transform on higher rank matrix domains is still open. It seems that the approach in [8] and 23] for compact symmetric domains combined with the results in this paper could lead to some interesting results. As is mentioned in Remark 3.3 the functions $|\mathrm{CH}(x)|^{\delta}$ for certain $\delta$ constitute the kernel of the Berezin transform which commutes with invariant differential operators (due to an explicit formula for $|\mathrm{CH}(x)|^{\delta}$ involving certain Bergman kernels) whereas it is not known if it is true for the operator with the kernel $|\mathrm{SH}(x)|^{\delta}$ (and we think it is not). It would be interesting to understand the precise relations between the two operators.

Remark 5.5. The reader may have noticed that Proposition 4.2 is proved by using Lemma 4.1, which is only proved (to the knowledge of the author) for root systems of symmetric cones. We conjecture that Lemma 4.1 is true for general non-negative multiplicity $a$ and for general non-symmetric functions $f$. It is not difficult to prove 
the analytic continuation of $\mathcal{G}_{\lambda}$ for root systems of type $A$ using the Cayley-Capelli type identities with the Dunkl operators [26]. (This will then give a refinement of Theorem 3.4 showing that $\Gamma_{a}\left(\frac{\delta-1+\iota+2 b}{2}+\frac{a}{2}(r-1)+1\right)|\mathrm{SH}(t)|^{\delta}$ has an analytic continuation.) However it seems that to prove the result on the Dirac distribution some more work on the Laplace transform 2 on the root system of type $A$ has to be done.

\section{REFERENCES}

[1] J.-P. Anker and L. Ji, Heat kernel and Green function estimates on noncompact symmetric spaces, Geom. Funct. Anal. 9 (1999), no. 6, 1035-1091. MR.1736928 (2001b:58038)

[2] T.H. Baker and P.J. Forrester, Non-symmetric Jack polynomials and intergral kernels, Duke Math. J. 95 (1998), no. 1, 1-50. MR.1646546 (2000b:33006)

[3] I. Cherednik, Inverse Harish-Chandra transform and difference operators, Internat. Math. Res. Notices (1997), no. 15, 733-750. MR1470375 (99d:22018)

[4] J. Faraut and A. Koranyi, Analysis on symmetric cones, Oxford University Press, Oxford, 1994. MR 1446489 (98g:17031)

[5] R. Gangolli and V. S. Varadarajan, Harmonic analysis of spherical functions on real reductive groups, Ergebnisse der Mathematik und ihrer Grenzgebiete [Results in Mathematics and Related Areas], vol. 101, Springer-Verlag, Berlin, 1988. MR954385 (89m:22015)

[6] I. M. Gel'fand, M. I. Graev, and R. Roşu, The problem of integral geometry and intertwining operators for a pair of real Grassmannian manifolds, J. Operator Theory 12 (1984), no. 2, 359-383. MR757440 (86c:22016)

[7] F. B. Gonzalez and T. Kakehi, Pfaffian systems and Radon transforms on affine Grassmann manifolds, Math. Ann. 326 (2003), no. 2, 237-273. MR.1990910 (2004f:53093)

[8] E. Grinberg and B. Rubin, Radon inversion on Grassmannians via Gårding-Gindikin fractional integrals, Ann. of Math. (2) 159 (2004), no. 2, 783-817. MR2081440 (2005f:58042)

[9] E. L. Grinberg, Radon transforms on higher Grassmannians, J. Differential Geom. 24 (1986), no. 1, 53-68. MR857375 (87m:22023)

[10] G. Heckman and H. Schlichtkrull, Harmonic analysis and special functions on symmetric spaces, Perspectives in Mathematics, vol. 16, Academic Press Inc., San Diego, CA, 1994. MR $1313912(96 \mathrm{j}: 22019)$

[11] G. J. Heckman, Root systems and hypergeometric functions. II, Compositio Math. 64 (1987), no. 3, 353-373. MR.918417 (89b:58192b)

[12] G. J. Heckman and E. M. Opdam, Root systems and hypergeometric functions. I, Compositio Math. 64 (1987), no. 3, 329-352. MR918416 (89b:58192a)

[13] S. Helgason, Differential geometry and symmetric spaces, Academic Press, New York, London, 1978. MR0145455 (26:2986)

[14] S. Helgason, The Radon transform, second ed., Progress in Mathematics, vol. 5, Birkhäuser Boston Inc., Boston, MA, 1980. MR573446 (83f:43012)

[15] S. Helgason, Groups and geometric analysis, Academic Press, New York, London, 1984. $\operatorname{MR} 754767(86 \mathrm{c}: 22017)$

[16] Sigurdur Helgason, The surjectivity of invariant differential operators on symmetric spaces. I, Ann. of Math. (2) 98 (1973), 451-479. MR0367562 (51:3804)

[17] T. Kakehi, Integral geometry on Grassmann manifolds and calculus of invariant differential operators, J. Funct. Anal. 168 (1999), no. 1, 1-45. MR.1717855 (2000k:53069)

[18] O. Loos, Bounded symmetric domains and Jordan pairs, University of California, Irvine, 1977.

[19] E. Opdam, Harmonic analysis for certain representations of graded Hecke algebras, Acta Math. 175 (1995), no. 1, 75-121. MR1353018 (98f:33025)

[20] E. Ournycheva and B. Rubin, The Radon transform of functions of matrix argument, preprint.

[21] B. Rubin, Radon, cosine and sine transforms on real hyperbolic space, Adv. Math. 170 (2002), 206-223. MR.1932329 (2004b:43007)

[22] R. Schimming and H. Schlichtkrull, Helmholtz operators on harmonic manifolds, Acta Math. 173 (1994), no. 2, 235-258. MR1301393 (96g:58175) 
[23] G. Zhang, Radon transform on real, complex and quaternionic Grassmannians, Duke Math. J., 138 (2007), no. 1, 137-160. MR2309157(2008c:44002)

[24] _ Spherical transform and Jacobi polynomials on root systems of type BC, Intern. Math. Res. Notices, 2005, no. 51. 3169-3189. MR2187504 (2006i:33014)

[25] _ Berezin transform on compact Hermitian symmetric spaces, Manuscripta Math. 97 (1998), no. 3, 371-388. MR.1654800 (2000c:22013)

[26] Branching coefficients of holomorphic representations and Segal-Bargmann transform, J. Funct. Anal. 195 (2002), 306-349. MR1940358 (2004f:32026)

Department of Mathematics, Chalmers University of Technology and Göteborg UniVERsity, Göteborg, SWeden

E-mail address: genkai@math.chalmers.se 